\title{
ON LOG CANONICAL INVERSION OF ADJUNCTION
}

\author{
CHRISTOPHER D. HACON
}

\section{To $V$. Shokurov on his sixtieth birthday}

\begin{abstract}
We prove a result on the inversion of adjunction for log canonical pairs that generalizes Kawakita's result to log canonical centers of arbitrary codimension.
\end{abstract}

The minimal model program is an ambitious program that aims to generalize to higher dimensional varieties many of the results in the classification of surfaces obtained by the Italian school of Algebraic Geometry in the early 20-th century. Log canonical singularities are the largest class of singularities for which the minimal model program is expected to hold. Let $(X, \Delta)$ be a pair consisting of a normal variety $X$ and an effective $\mathbb{Q}$-divisor $\Delta=\sum \delta_{i} \Delta_{i}$ such that $K_{X}+\Delta$ is $\mathbb{Q}$ Cartier. Consider a $\log$ resolution of $(X, \Delta)$ i.e. a proper birational morphism $\mu$ : $Y \rightarrow X$ such that $Y$ is smooth, $\operatorname{Exc}(\mu)$ is a divisor and $\mu_{*}^{-1} \Delta+\operatorname{Exc}(\mu)$ has simple normal crossings. Write $K_{Y}+\Delta_{Y}=\mu^{*}\left(K_{X}+\Delta\right)$, then $\Delta_{Y}=\sum b_{i} B_{i}$ is uniquely determined and $(X, \Delta)$ is $\log$ canonical (resp. $k l t)$ if $b_{i} \leq 1$ (resp. $b_{i}<1$ ) for all $i$. Similarly $(X, \Delta)$ is plt if $0 \leq \delta_{i} \leq 1$ and for all $\log$ resolutions $\mu: Y \rightarrow X$ we have $b_{i}<1$ for all $i$ such that $B_{i}$ is $\mu$-exceptional. Suppose that $\Delta=S+B$ where $S$ is a prime divisor and $\nu: S^{\nu} \rightarrow S$ is the normalization, then there is a uniquely defined $\mathbb{Q}$-divisor $\operatorname{Diff}(B)$ on $S^{\nu}$ such that $\left.\left(K_{X}+S+B\right)\right|_{S^{\nu}}=K_{S^{\nu}}+\operatorname{Diff}(B)$ (cf. Section 16 of [Kollár et al.]). Note that if $S^{\prime}$ is the strict transform of $S$ on $Y$ and $\bar{\mu}: S^{\prime} \rightarrow S^{\nu}$ is the induced morphism, then $\operatorname{Diff}(B)=\bar{\mu}_{*}\left(\left.\left(\Delta_{Y}-S^{\prime}\right)\right|_{S^{\prime}}\right)$.

It is of fundamental importance in the minimal model program to relate the singularities of the pair $(X, S+B)$ to those of the pair $\left(S^{\nu}, \operatorname{Diff}(B)\right)$. If we know that the pair $(X, S+B)$ is $\log$ canonical (resp. plt), then it is easy to see that the pair $\left(S^{\nu}, \operatorname{Diff}(B)\right)$ is also log canonical (resp. klt). This process is known as adjunction. Inverse of adjunction on the other hand is the process of deducing information about the singularities on the ambient variety $(X, S+B)$ from information on the singularities of the divisor $\left(S^{\nu}, \operatorname{Diff}(B)\right)$. These results are much more subtle and useful. It is known that if $\left(S^{\nu}, \operatorname{Diff}(B)\right)$ is klt, then $(X, S+B)$ is plt on a neighborhood of $S$ (cf. KKollár et al., 17.6]) and by a more recent result of Kawakita, that if $\left(S^{\nu}, \operatorname{Diff}(B)\right)$ is lc, then $(X, S+B)$ is lc on a neighborhood of $S$ (cf. Kawakita07). The proof of both results heavily relies on Kawamata-Viehweg vanishing.

Date: February 3, 2012.

The author was partially supported by NSF research grant no: 0757897 . 
The purpose of this short note is to give a proof of a generalization of Kawakita's theorem on the inversion of adjunction to higher co-dimensional subvarieties. Our proof is based on the results of [BCHM10] and recovers a new proof of Kawakita's theorem. Our argument closely follows ideas of Shokurov (cf. Shokurov93]), but avoids the use of the minimal model program for log canonical pairs. Note moreover that there are some similarities with this proof and the arguments of [Kawakita07] and [Kollár et al.]. See also [Schwede09] for a related result in characteristic $p>0$.

Before stating the main theorem we must introduce some notation. Let $(X, \Delta)$ be a pair, then a subvariety $V \subset X$ is a log canonical center if there is a log resolution $\mu: Y \rightarrow X$ such that $K_{Y}+\Delta_{Y}=\mu^{*}\left(K_{X}+\Delta_{X}\right)$ where $\Delta_{Y}=\sum b_{i} B_{i}$ and $\max \left\{b_{i} \mid \mu\left(B_{i}\right)=V\right\}=1$. Recall that in this case, $(X, \Delta)$ is log canonical on a neighborhood of the generic point of $V$ (cf. [Kollár et al., 17.1.1]). We will denote by $S$ a component $B_{i}$ as above, such that $b_{i}=1$ and $\mu\left(B_{i}\right)=V$. Let $\nu: W \rightarrow V$ be a birational morphism from a normal variety $W, \Delta_{S}:=\left.\left(\Delta_{Y}-S\right)\right|_{S}$, and assume that $\bar{\mu}: S \rightarrow W$ is a morphism. Then we define an effective $\mathbb{Q}$-divisor $B_{W}=\sum\left(1-t_{i}\right) P_{i}$ on $W$ as follows: for any codimension 1 point $P_{i}$ on $W$, let

$$
t_{i}=\sup \left\{\tau \mid\left(S, \Delta_{S}+\tau \bar{\mu}^{*} P_{i}\right) \text { is lc over } \eta_{P_{i}}\right\}
$$

where $\eta_{P_{i}}$ denotes the generic point of $P_{i}$. Note that the $t_{i}$ are rational numbers (positive or negative). It is known that (cf. Ambro99)

(1) The numbers $t_{i}$ are independent of the $\log$ resolution $\mu: Y \rightarrow X$ and of the choice of the divisor $S$.

(2) If $W=V^{\nu}$ is the normalization of $V$, then $1-t_{i} \geq 0$ for all $P_{i} \in V^{\nu}$, and the strict inequality only holds for finitely many codimension 1 points $P_{i} \in W$.

(3) If $S$ is the only component of $\Delta_{Y}$ of coefficient 1 , then $\left(W, B_{W}\right)$ is klt.

(4) When $\operatorname{dim} V=\operatorname{dim} X-1$, we let $S$ be the strict transform of $V$ and we have $B_{V^{\nu}}=\operatorname{Diff}(\Delta-V)$ where $V^{\nu} \rightarrow V$ is the normalization.

(5) If $\eta: W^{\prime} \rightarrow W$ is a birational morphism of normal varieties, then $\eta_{*} B_{W^{\prime}}=$ $B_{W}$ so that we have a b-divisor $\mathbf{B}=\mathbf{B}(V ; X, \Delta)$ defined by $\mathbf{B}_{W}=B_{W}$ (see [Corti07] for the definition and properties of $\mathbf{b}$-divisors).

(6) If $S \rightarrow W$ satisfies the standard normal crossing assumptions of [Kollár07, 8.3.6], then $\mathbf{B}$ descends to $W$ in the sense that for any birational morphism $\eta: W^{\prime} \rightarrow W$, we have $\eta^{*}\left(K_{W}+\mathbf{B}_{W}\right)=K_{W^{\prime}}+\mathbf{B}_{W^{\prime}}$.

(7) If $\left(W, B_{W}\right)$ is sub-log canonical (i.e. if $t_{i} \geq 0$ for any $P_{i} \in W$ ) for any sufficiently high model (or equivalently for any model $W$ such that $S \rightarrow$ $W$ satisfies the standard normal crossing assumptions) then we say that $\left(V^{\nu}, \mathbf{B}\right)$ is log canonical. Note that by the Base Change Conjecture (cf. [Ambro99, Conjecture 5]) it is expected that $K_{V^{\nu}}+B_{V^{\nu}}$ is $\mathbb{Q}$-Cartier and $\mathbf{B}$ descends to $V^{\nu}$ in which case $\left(V^{\nu}, \mathbf{B}\right)$ is $\log$ canonical if and only if $\left(V^{\nu}, B_{V^{\nu}}\right)$ is $\log$ canonical. 
We will prove the following generalization of Kawakita's result:

Theorem 0.1. Let $V$ be a log canonical center of a pair $\left(X, \Delta=\sum \delta_{i} \Delta_{i}\right)$ where $0 \leq \delta_{i} \leq 1$. Then $(X, \Delta)$ is $\log$ canonical on a neighborhood of $V$ if and only if $\left(V^{\nu}, \mathbf{B}(V ; X, \Delta)\right)$ is log canonical.

Proof. If $(X, \Delta)$ is log canonical on a neighborhood of $V$, then it is well known and easy to see that $\left(V^{\nu}, \mathbf{B}(V ; X, \Delta)\right)$ is $\log$ canonical. Therefore, we will assume that $\left(V^{\nu}, \mathbf{B}(V ; X, \Delta)\right)$ is $\log$ canonical and we will prove that $(X, \Delta)$ is log canonical on a neighborhood of $V$. Let $\mu: Y \rightarrow X$ be a dlt model (cf. [KK10, 3.1]) of $(X, \Delta)$, so that if we write $\mu^{*}\left(K_{X}+\Delta\right)=K_{Y}+\Delta_{Y}$, then

(1) $Y$ is $\mathbb{Q}$-factorial,

(2) $\Delta_{Y}=\sum b_{i} B_{i} \geq 0$

(3) $\left(Y, \Delta_{Y}^{\prime}=\sum_{b_{i}<1} b_{i} B_{i}+\sum_{b_{i}>1} B_{i}\right)$ is dlt, and

(4) every exceptional divisor appears in $\Delta_{Y}^{\prime}$ with coefficient $\geq 1$.

We may assume that $\Delta_{Y}^{\prime}=S+\Gamma$ where $S$ is an irreducible component of $\Delta_{Y}^{=1}=$ $\sum_{b_{i}=1} B_{i}$ that dominates $V$ and $\Sigma=\Delta_{Y}-S-\Gamma$. Note that $f(\Sigma) \not \supset V$. We now fix a sufficiently ample divisor $H$ on $Y$ and we run the $\left(K_{Y}+S+\Gamma\right)$-MMP with scaling of $H$ over $X$ (cf. [BCHM10, 3.10]). Let $\phi_{i}: Y_{i} \rightarrow Y_{i+1}$ be the induced sequence of flips and divisorial contractions and let $\mu_{i}: Y_{i} \rightarrow X$ be the induced morphisms. For any divisor $G$ on $Y$ we let $G_{i}$ be its strict transform on $Y_{i}$. Then there is a non-increasing sequence of rational numbers $s_{i} \geq s_{i+1}$ which is either

- finite with $s_{N+1}=0$, or

- infinite with $\lim s_{i}=0$

such that $K_{Y_{i}}+S_{i}+\Gamma_{i}+s H_{i}$ is nef over $X$ for all $s_{i} \geq s \geq s_{i+1}$. For $i \geq i_{0}$, we may assume that each $\phi_{i}$ is a flip. By a well known discrepancy computation, we may also assume that $S_{i} \rightarrow S_{i+1}$ is an isomorphism in codimension 1 for all $i \geq i_{0}$ (cf. the arguments in the proofs of Step 1 and Step 2 of [Fujino07, 4.2.1]). For any $t>0$ there exists a $\mathbb{Q}$-divisor $\Theta_{t}$ on $Y$ such that $\Theta_{t} \sim_{\mathbb{Q}} \Gamma+t H$ and $\left(Y, S+\Theta_{t}\right)$ is plt. Note that if $t<s_{i}$, then $\left(Y_{i}, S_{i}+\Theta_{t, i}\right)$ is plt (this is because plt singularities are preserved by steps of the minimal model program).

Suppose that for some $i \geq 0$, we have $S_{i} \cap \Sigma_{i} \neq \emptyset$, then

$$
\left.\left(\mu_{i}^{*}\left(K_{X}+\Delta\right)\right)\right|_{S_{i}}=\left.\left(K_{Y_{i}}+S_{i}+\Gamma_{i}+\Sigma_{i}\right)\right|_{S_{i}}=K_{S_{i}}+\operatorname{Diff}\left(\Gamma_{i}+\Sigma_{i}\right)
$$

is not $\log$ canonical. Let $\bar{\mu}_{i}: S_{i} \rightarrow V^{\nu}$ be the induced morphism. We may replace $S_{i} \rightarrow V^{\nu}$ by a birational model $\widetilde{\mu}: \widetilde{S} \rightarrow W$ satisfying the standard normal crossing assumptions. If $g: \widetilde{S} \rightarrow S_{i}$ is the induced morphism and we write $K_{\widetilde{S}}+\Delta_{\widetilde{S}}=g^{*}\left(K_{S_{i}}+\operatorname{Diff}\left(\Gamma_{i}+\Sigma_{i}\right)\right)$, then there is a component of $\Delta_{\widetilde{S}}$ of coefficient $>1$. After possibly replacing $W$ by an appropriate birational model, we may assume that the image of this component is a codimension 1 point $P_{i} \in W$. But then $t_{i}<0$ so that $1-t_{i}>1$ and hence $\left(W, B_{W}\right)$ is not log canonical. This proves the theorem. 
Therefore, we will assume that $S_{i} \cap \Sigma_{i}=\emptyset$ for all $i \geq 0$, and we will derive a contradiction. Note that if this is the case, then any curve contained in $S_{i}$ intersects $\Sigma_{i}$ trivially and hence $\phi_{i}$ does not contract $S_{i}$. For any $m \gg 0$ such that $m \Sigma$ is an integral divisor, let $i \gg 0$ be the integer such that $s_{i}>\frac{1}{m} \geq s_{i+1}$. Notice that since

$$
H_{i}-m \Sigma_{i}-S_{i} \sim_{\mathbb{Q}, X} K_{Y_{i}}+\Theta_{\frac{1}{m}, i}+(m-1)\left(K_{Y_{i}}+S_{i}+\Gamma_{i}+\frac{1}{m} H_{i}\right)
$$

where $\left(Y_{i}, \Theta_{\frac{1}{m}, i}\right)$ is klt and $K_{Y_{i}}+S_{i}+\Gamma_{i}+\frac{1}{m} H_{i}$ is nef over $X$, then by KawamataViehweg vanishing (cf. [KM98, 2.70]), we have that $R^{1}\left(\mu_{i}\right)_{*} \mathcal{O}_{Y_{i}}\left(H_{i}-m \Sigma_{i}-S_{i}\right)=0$ and hence

$$
\left(\mu_{i}\right)_{*} \mathcal{O}_{Y_{i}}\left(H_{i}-m \Sigma_{i}\right) \rightarrow\left(\bar{\mu}_{i}\right)_{*} \mathcal{O}_{S_{i}}\left(H_{i}\right)
$$

is surjective. On the other hand, for $m \gg 0$, the subsheaves

$$
\left(\mu_{i}\right)_{*} \mathcal{O}_{Y_{i}}\left(H_{i}-m \Sigma_{i}\right)=\left(\mu_{i_{0}}\right)_{*} \mathcal{O}_{Y_{i_{0}}}\left(H_{i_{0}}-m \Sigma_{i_{0}}\right) \subset\left(\mu_{i_{0}}\right)_{*} \mathcal{O}_{Y_{i_{0}}}\left(H_{i_{0}}\right)
$$

are contained in $\mathcal{I}_{\mu_{i_{0}}\left(\Sigma_{i_{0}}\right)} \cdot\left(\mu_{i_{0}}\right)_{*} \mathcal{O}_{Y_{i_{0}}}\left(H_{i_{0}}\right)$. Since $V \cap \mu_{i_{0}}\left(\Sigma_{i_{0}}\right) \neq \emptyset$ and $S_{i_{0}} \rightarrow S_{i}$ is an ismorphism in codimension 1 , the induced homomorphism

$$
\left(\mu_{i}\right)_{*} \mathcal{O}_{Y_{i}}\left(H_{i}-m \Sigma_{i}\right) \rightarrow\left(\bar{\mu}_{i_{0}}\right)_{*} \mathcal{O}_{S_{i_{0}}}\left(H_{i_{0}}\right)=\left(\bar{\mu}_{i}\right)_{*} \mathcal{O}_{S_{i}}\left(H_{i}\right)
$$

is not surjective. This is the required contradiction.

\section{REFERENCES}

[Ambro99] F. Ambro, The Adjunction Conjecture and its applications. arXiv9903060v3

[BCHM10] C. Birkar, P. Cascini, C. Hacon, and J. Mckernan, Existence of minimal models for varieties of log general type, J. Amer. Math. Soc. 23 (2010), no. 2, 405-468.

[Corti07] A. Corti, 3-fold flips after Shokurov in Flips for 3-folds and 4-folds. Oxford Lecture Series in Mathematics and its Applications 35. Oxford University Press, Oxford (2007).

[Fujino07] O. Fujino, Special termination and reducion to pl-flips in Flips for 3-folds and 4-folds. Oxford Lecture Series in Mathematics and its Applications 35. Oxford University Press, Oxford (2007).

[Kawakita07] M. Kawakita, Inversion of adjunction on log canonicity. Invent. Math. 167 (2007), no. 1, 129-133.

[Kawamata98] Y. Kawamata, Subadjunction of log canonical divisors. II. Amer. J. Math. 120 (1998), no. 5, 893-899.

[Kollár et al.] J. Kollár et al., Flips and abundance for algebraic threefolds, Société Mathématique de France, Paris, 1992, Papers from the Second Summer Seminar on Algebraic Geometry held at the University of Utah, Salt Lake City, Utah, August 1991, Astérisque No. 211 (1992).

[KM98] J. Kollár, S. Mori, Birational geometry of algebraic varieties. With the collaboration of C. H. Clemens and A. Corti. Translated from the 1998 Japanese original. Cambridge Tracts in Mathematics, 134. Cambridge University Press, Cambridge, 1998.

[Kollár07] J. Kollár, Kodaira's canonical bundle formula and adjunction. Flips for 3-folds and 4-folds, 134-162, Oxford Lecture Ser. Math. Appl., 35, Oxford Univ. Press, Oxford, 2007 
[KK10] J. Kollár, S. Kovács, Log canonical singularities are Du Bois. J. Amer. Math. Soc. 23 (2010), no. 3, 791-813.

[Schwede09] K. Schwede, F-adjunction. Algebra Number Theory 3 (2009), no. 8, 907950.

[Shokurov93] V. V. Shokurov, Semi-stable 33-fold flips. Izv. Ross. Akad. Nauk Ser. Mat. 57 (1993), no. 2, 165-222.

Department of Mathematics, University of Utah, 155 South 1400 East, JWB 233, Salt Lake City, UT 84112, USA

E-mail address: hacon@math.utah.edu 